\title{
Wetting enhanced by water adsorption in hygroscopic plantlike materials
}

\author{
Meng Zhou, ${ }^{1}$ Sabine Caré, ${ }^{1}$ Andrew King, ${ }^{2}$ Denis Courtier-Murias, ${ }^{1}$ Stéphane Rodts,,${ }^{1, *}$ Gaétan Gerber, ${ }^{1}$ Patrick Aimedieu, ${ }^{1}$ \\ Marie Bonnet, ${ }^{1}$ Michel Bornert, ${ }^{1}$ and Philippe Coussot $\oplus^{1, \dagger}$ \\ ${ }^{1}$ Université Paris-Est, Laboratoire Navier (UMR 8205), CNRS, Ecole des Ponts ParisTech, IFSTTAR, 77455 Marne-la-Vallée, France \\ ${ }^{2}$ Synchrotron SOLEIL, L'Orme des Merisiers, 91190 Saint-Aubin, France
}

(Received 30 September 2019; published 20 December 2019)

\begin{abstract}
Water inside hygroscopic porous media such as plantlike systems can be found either freely penetrating in capillaries or absorbed into the solid phase (bound water). Here we demonstrate that the wetting properties (contact angle) of liquid along cell walls significantly depend on the amount of bound water absorbed: a change from poor to good wetting is observed when cell walls are saturated with bound water, which allows liquid displacement. We further show that this process is operative in hydrogels, suggesting that this might be a general property of porous hygroscopic systems. As a consequence, imbibition dynamics is controlled by water adsorption and diffusion in the walls, and even if the dynamics of capillary imbibition is strongly damped (by several orders of magnitude), water can freely climb over significant heights as long as sufficient water has been adsorbed into cell walls or in other hygroscopic walls. Under these conditions, the imbibition process in such systems is not described by the standard model but is analogous to the propagation of a front of solidification in a liquid. This process might contribute to the regulation of water absorption in unsaturated wood, allowing it to store available bound water in progressively higher depths, instead of leaving free water rapidly flow through it. Such a mechanism may be explored to design porous materials with tunable liquid adsorption timings for pharmaceutical or chemical engineering applications.
\end{abstract}

DOI: 10.1103/PhysRevResearch.1.033190

\section{INTRODUCTION}

Wood material, one of the oldest materials in human history, is being used increasingly in construction and in various other applications thanks to its remarkable mechanical, thermal, and hygroscopic properties. Water transfers as vapor or liquid strongly influence such properties and the structural alterations of the material with time, but the physical mechanisms governing such processes are still not fully understood. On the natural side, water is vital to plants. It is transported or stored in liquid state inside capillaries or as bound water adsorbed into cell walls [1]. These processes rely on a subtle hydraulic system that could be inspirational to develop new materials with original properties. The mechanism of transpiration in living plants is described according to the cohesion-tension theory, in which evaporation induces a water loss and thus a negative pressure pulls out liquid from the soil and moves it up in the xylem [1-4]. On the other hand, the coexistence of both air and liquid inside the vessels is a source of more complex behavior and problems such as cavitation (leading to embolism), that can stop circulation of liquid in vessels [5-7], or imbibition-drying cycles which may induce

\footnotetext{
${ }^{*}$ Deceased

${ }^{\dagger}$ Corresponding author: philippe.coussot@ifsttar.fr

Published by the American Physical Society under the terms of the Creative Commons Attribution 4.0 International license. Further distribution of this work must maintain attribution to the author(s) and the published article's title, journal citation, and DOI.
}

damages (swelling, fractures) in wooden construction [8,9]. Such transports are generally considered as being governed by laws similar to those in a porous medium with neutral structure. However, the permeability, although in principle an intrinsic parameter, was found to vary with the sample length [10-14], which suggests that some effect might affect the basic liquid transport in wood. Moreover, it was shown that subtle hydraulic mechanisms between adjacent vessels can foster water refilling in embolized xylems vessels $[15,16]$.

Recently, it was suggested that some interplay between bound and free water could affect $[17,18]$ the imbibition process. More precisely, it was shown that the capillary imbibition process of water in a poplar sample is strongly slowed down with regards to the dynamics expected from the theory relying on liquid-solid interactions and wood structure [18]. In contrast, oil imbibition is much faster (typically by three orders of magnitude) and its dynamics well described by the standard theory. Finally, it was shown that this extremely slow imbibition of water is associated with the transport of water in the form of bound water at larger distance than free water, suggesting that this bound water could control the free water advance [18] but the origin of this effect is not understood.

Here, we demonstrate that this effect finds its origin in a strong modification of the wetting properties of the wood depending on its bound water content. We start by showing that the effect of damped imbibition dynamics described above is general in hardwoods. Then we focus on one typical hardwood, a hornbeam, for which we check through MRI (magnetic resonance imaging) that bound water propagates farther than free water along the longitudinal (imbibition) axis. 3D Synchrotron x-ray microcomputed tomography 
images show that the wetting characteristics (contact angle) are strongly different depending on the amount of bound water adsorbed in cell walls. The generality of this effect is then demonstrated by reproducing it with hydrogels, a "synthetic tree" mimicking material used in the study of wood transpiration and cavitation processes [4,5]. More precisely, placed in contact with free water, the contact angle will differ from about $90^{\circ}$ only when the pore walls have been filled with bound water. As a result, spontaneous imbibition is considerably retarded (by several orders of magnitude). We then show that under these conditions the imbibition is analogous to the propagation of a front of solidification in a liquid. This process might contribute to a regulation of water absorption in plantlike systems after some drying period, as it allows the storage of available water molecules as bound water in progressively higher depths, instead of leaving free water rapidly flow through the structure. Overall, our findings extend our understanding of water transport within porous materials and set the foundations for the development of bioinspired porous materials able to absorb liquid with a tunable timing for chemical and pharmaceutical applications.

\section{RESULTS}

\section{A. Paradoxical capillary Imbibition in hardwoods}

Wood structure is essentially made of elongated hollow cellular units directed along the direction of the tree trunk (called longitudinal direction), and along which a liquid (i.e., free water) can move in lumens, surrounded by a solid structure able to adsorb water (i.e., bound water) $[3,10]$. Hardwoods are basically made of long (hollow) dead-end vessels whose diameter ranges from a few tens to about one hundred of microns depending on species (see Appendix A), in a solid structure containing elongated enclosed fibers (with a radius around a few microns) able to store some free water. The vessels can ensure a significant liquid transport because of their large hydraulic radius and their length which sometimes can reach several meters [3]. The basic experiment for understanding liquid uptake consists of taking a wood sample without free water (either in vessels or fibers), putting it in contact with a liquid source and observing the imbibition process in the longitudinal direction [Fig. 1(a)]. Under such conditions we expect an imbibition process basically governed by capillary effects, i.e., the liquid wets the vessel wall with some contact angle (thus forming a meniscus) which induces a pressure drop which pulls the fluid upwards against gravity. As already observed for other wood types with different techniques [19-23], we can observe with MRI (see Appendix C) a progressive penetration of water from the sample bottom, preferentially between growth rings [Fig. 1(b)]. The climbing dynamics varies with growth rings, due to the structure heterogeneity resulting from variable growth related to environmental condition fluctuations. A first approach to quantify imbibition is to follow the sample mass variations with time and compute the average characteristic height $h^{*}=$ $\Omega / S$ (with $\Omega$ the absorbed liquid volume and $S$ the sample cross-section) as a function of time [Fig. 1(c)].

Imbibition tests have been performed on two ring-porous woods (oak, ash), a diffuse-porous (hornbeam) and a semidiffuse-porous (intermediate) wood (poplar) (see properties in (a)

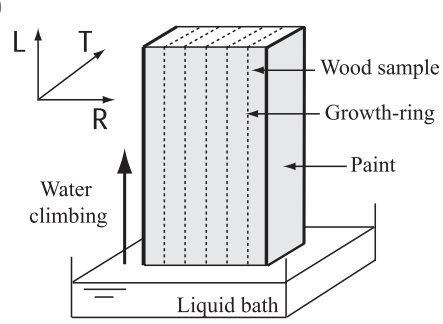

(b)
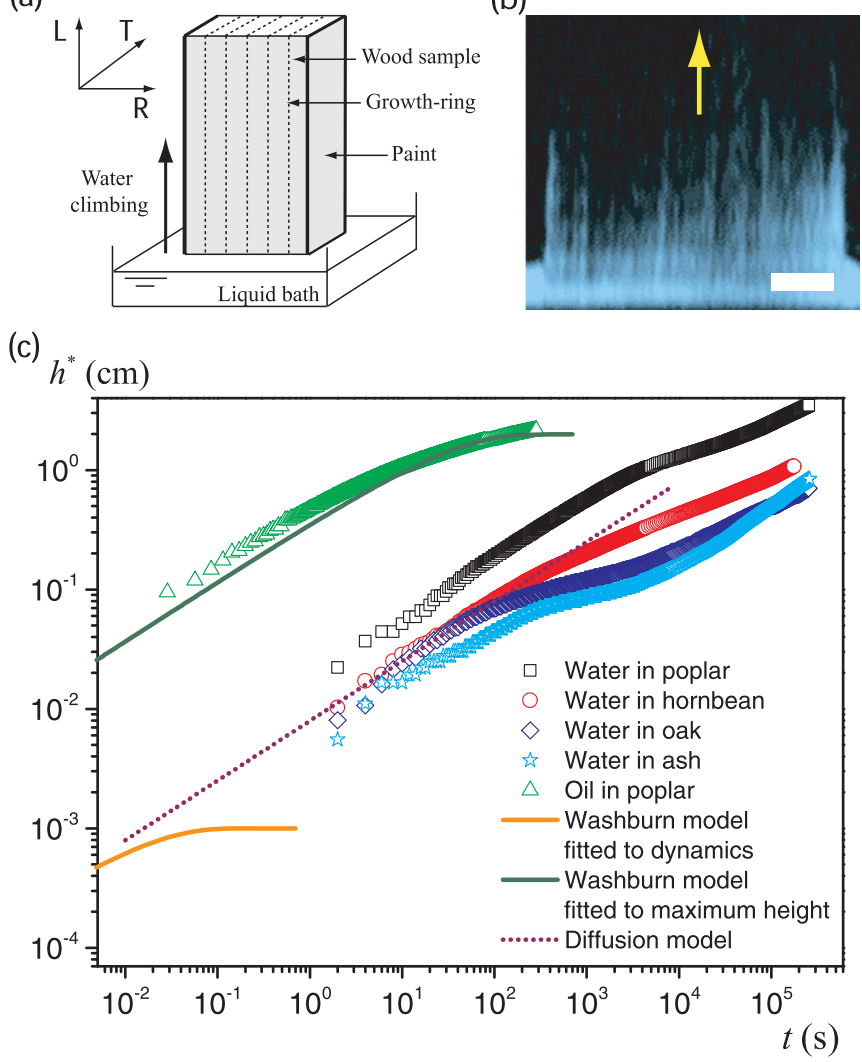

(c)

FIG. 1. (a) Principle of the imbibition experiment, showing the orientation of the wood sample, whose vertical faces have been sealed with paint. For all our tests the sample size was $4(\mathrm{R}) \times 2(\mathrm{~T}) \times$ $10(\mathrm{~L}) \mathrm{cm}^{3}$ where $\mathrm{R}$ and $\mathrm{T}$ are the radial and tangential directions and $\mathrm{L}$ the longitudinal direction (vertical tree axis). (b) Typical 2D MRI image (sample width $4 \mathrm{~cm}$ ) taken during imbibition in a hornbeam sample (free water in light blue, bound water and dry wood are not visible in such an image). The yellow arrow indicates the direction of water movement. Scale bar: $1 \mathrm{~cm}$. (c) Absorbed water volume per transversal cross-section area as a function of time for different wood types. The continuous lines correspond to the complete Washburn model [Eq. (1)] fitted to either the slope at short time (orange curve), or approximately the maximum level reached for hornbeam data (green curve). The purple dotted line (of slope $\frac{1}{2}$ ) corresponds to the predicted water amount according to the diffusion model [Eq. (6)]. The (green) triangles correspond to the same test for a poplar sample (data from Ref. [18]) in contact with a silicone oil $\left(\sigma_{1}=0.02 \mathrm{~N} \mathrm{~m}^{-1}\right.$; $\mu_{1}=20 \mathrm{mPa}$ s), whose time was normalized by $\mu \sigma_{1} / \mu_{1} \sigma$ in order to obtain imbibition data comparable to those of water within the frame of a Washburn approach.

Appendix A), which cover the three typical types of vessel structure of hardwood. For all tested species we observe an imbibition process at first sight consistent with a standard Washburn process [24,25] since the imbibition curves roughly tend to follow a square root of time variation [Fig. 1(c)] This conclusion is in line with previous observations from imbibition tests on wood, generally carried out in a much narrower range of times [22,26].

A quantitative analysis of the data with the help of the Washburn model is nevertheless possible. The capillary ascent of a liquid in a cylindrical conduit (of radius $R$ ) is driven by 
the capillary pressure defined as $p=-2 \sigma \cos \theta / R$, where $\sigma$ is the surface tension (we use $\sigma=0.07 \mathrm{~N} \mathrm{~m}^{-1}$ for water) and $\theta$ the contact angle [27], damped by gravity effects leading to a pressure gradient $\rho g$, where $\rho$ is the fluid density and $g$ the gravity. Assuming a laminar flow in the vessel, the pressure drop $(\Delta p)$ over the height $h$ of liquid in the conduit is also related to the flow characteristics via the Poiseuille law, which is expressed by $\Delta p / h=8 \mu V / R^{2}$, where $\mu$ is the liquid viscosity $\left(10^{-3} \mathrm{Pas}\right.$ for water) and $V=d h / d t$ is the mean flow velocity through the vessel. Finally, the liquid ascent in the conduit is described by

$$
8 \mu V / R^{2}=2 \sigma \cos \theta / h R-\rho g .
$$

According to this equation, in a first regime (short times) controlled by capillary effects the height of liquid $h$ is defined by

$$
h=\sqrt{K t}
$$

with a coefficient $K=\sigma R \cos \theta / 2 \mu$. In a second regime (long times) controlled by gravity effects, $h$ tends to a plateau determined by

$$
h_{\max }=2 \sigma \cos \theta / \rho g R=4 \mu K / \rho g R^{2} .
$$

If we assume that the wood samples are essentially made of simple straight conduits (vessels) dispersed in a solid phase (leading to an apparent porosity $\varepsilon$ ) and parallel to the longitudinal direction, this model applies with $h=h^{*} / \varepsilon$. Then, since we know the main geometrical characteristics of the hydraulic system, the fundamental unknown of the problem is the contact angle, which may be fitted to either the dynamics or the maximum height of ascent. However, no consistent fitting, even approximate, may be found.

Indeed, let us consider the observed coefficient for the dynamics in the first (square-root) regime in our experiments (i.e., $K \approx 7 \times 10^{-8} \mathrm{~m}^{2} \mathrm{~s}^{-1}$ ). The above expression for $h_{\text {max }}$ implies that the maximum height of ascent should be, e.g. for hornbeam, about $30 \mu \mathrm{m}$ (which implies $\theta \approx 90^{\circ}$ ), in strong contradiction with our data showing that water goes on climbing beyond one centimeter [Fig. 1(c)]. Conversely, if we assume wetting properties in agreement with the (approximate) observed $h_{\max }$ value, which implies $\theta \approx 82^{\circ}$ for hornbeam, the model predicts a much larger $K$ value, and consequently a much faster penetration dynamics than observed [see Fig. 1(c)], typically by a factor 1000 for hornbeam and even larger for the other species which have larger average vessel diameters (see Appendix A). Thus, we observe a much too slow or, conversely, a much too high imbibition than expected from a simple capillary (Washburn) imbibition dynamics. Note that here the maximum height was roughly estimated from the maximum height observed within the duration of the tests in Fig. 1(c). Such value constitutes a lower bound of the effective maximum height, which is sufficient for the demonstration of the paradox.

A previous quantitative physical analysis already emphasized a time of imbibition several orders of magnitude larger than expected from the permeability deduced from the wood structure [14]. On the one hand, to explain these results, structures made of a network of channels with connections with a probability of passage [28,29], or with a double permeability [14], were suggested, which are nevertheless not fully consistent with the effective structure of hardwood. On the other hand, a dynamics differing from that expected from the standard Washburn process has been observed for bi-porous systems [30-33] and other peculiar effects were observed with specific pore shapes [25,34,35]. Among these materials were cellulose sponges [31,32] made of macropores (few millimeters) connected by micropores (few micrometers), and a solid structure formed of sheets able to adsorb water so that the structure swells when in contact with water. The late stage of water imbibition dynamics is slowed down by micropore deformation and merging, resulting from deformations of the hygroscopic structure [32]. However, this effect is much weaker (less than one order of magnitude) than that observed here, and hardwoods are a priori simpler systems (straight continuous vessels). In addition, this paradox cannot simply be explained by some loss of water amount in the walls and fiber lumens, which surround the vessels. Since the material can absorb up to typically 1.5 times the vessel volume, a water loss in this structure cannot induce a rate of imbibition slower than expected by a factor of three orders of magnitude.

Alternatively, we could suspect that, due to some structure features not apparent from microscopy, the pressure loss is much larger than assumed above considering vessels as straight cylindrical conduits. However, the good agreement of the data for oil with the Washburn prediction (see Fig. 1(c) and Ref. [18] for other oil viscosities) demonstrates that such extra pressure loss is negligible (as it would act in the same way for any liquid type). More generally, the consistency of oil data with the standard Washburn model predictions, which contrasts with the paradoxical results for water, confirms our appreciation of the main structure trends of the hydraulic network from microscopy, which suggests that the observed effect is specifically due to the affinity of water with wood. More precisely, this implies that the presence of bound water should have an impact on the wetting properties.

\section{B. Imbibition by propagation of planar air-liquid interfaces}

In order to find out the origin of this phenomenon, we need to clarify the wetting properties of wood which play a fundamental role in a standard capillary imbibition process. A simple measurement of the contact angle from the shape of a water droplet lying over the external sample surface is hardly relevant as it may be significantly affected by liquid penetration through the porosity or by the roughness of the surface. The most straightforward measurement consists to look directly at the wetting characteristics inside the wood vessels. In that aim we observe the water distribution at the micron-scale, from 3D Synchrotron X-ray microcomputed tomography images of the hornbeam sample (see Appendix B). From a detailed inspection of the transitions of grey level, resulting from different signal attenuations, along the different directions inside the vessels, we can clearly identify the position and shape of the air-liquid interfaces.

A small wood piece $\left[1.75(\mathrm{R}) \times 1.75(\mathrm{~T}) \times 6(\mathrm{~L}) \mathrm{mm}^{3}\right]$ initially conditioned at $54 \% \mathrm{RH}$ is placed in contact with a water bath, i.e., its bottom is plunged in water. After about ten minutes we look at the water distribution in the vicinity of the sample (vertical) L edges in contact with water, where bound or free water could rather easily penetrate from almost 

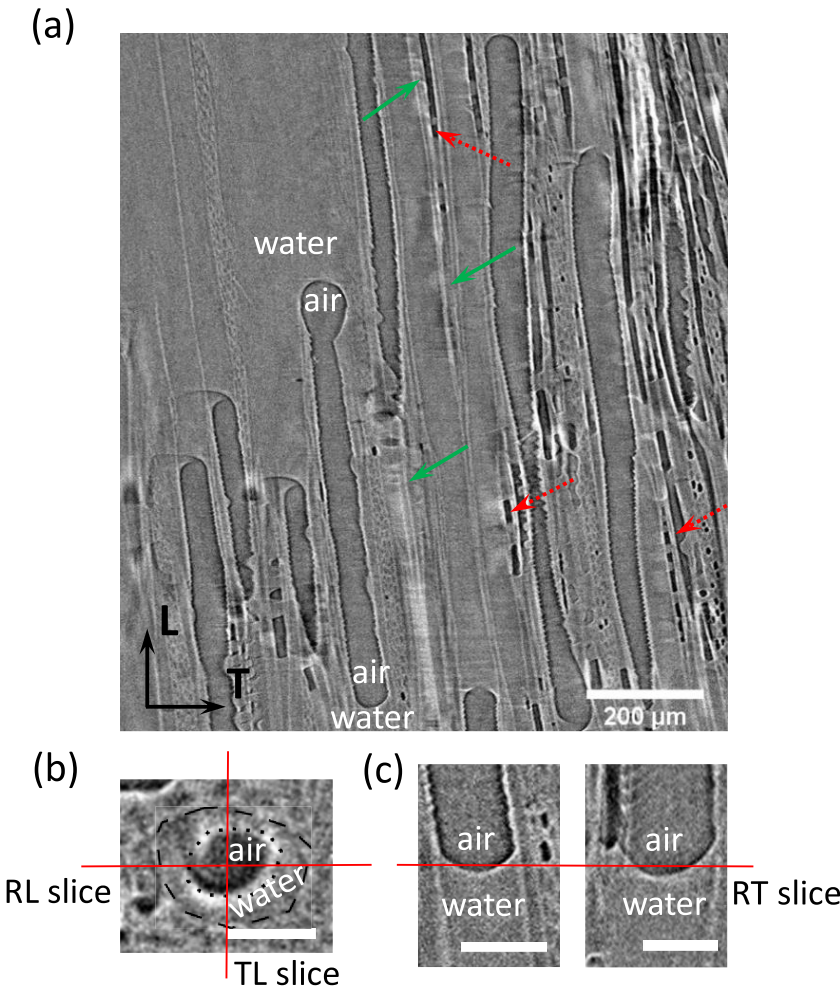

RT slice

\section{TL slice RL slice}

FIG. 2. Air-liquid interfaces in different directions inside vessels, along the vertical edges of a wood sample immersed in water. (a) Micrograph of a tangential-longitudinal (TL) section (at a distance of $100 \mu \mathrm{m}$ from the vertical edge) in a hornbeam sample after about $10 \mathrm{~min}$ in contact with water (without paint). Fibers are filled (green arrows) or partially filled (red arrows) with free water. (b) Aspect of a typical interface formed between air bubbles and water inside hornbeam wood vessels imaged at the transversal plane (RT slice) at the point where the radial, tangential and longitudinal planes intersect perpendicularly [see Fig. 1(a) for details]. The water/air meniscus is indicated by dotted lines and the vessel wall by dashed lines. (c) Tangential-longitudinal (TL) and radial-longitudinal (RL) cross-sectional images of an air-liquid interface formed in these samples. Scale bars are (a) 200 and [(b) and (c)] $50 \mu \mathrm{m}$.

all sides. Trapped air bubbles (Fig. 2) then allow to see that the air-liquid interfaces are significantly curved with a contact angle around $30^{\circ}$. Such a shape is consistent with the observation of menisci in leave vessels $[15,16]$. The point is that with such wetting properties, we would expect an imbibition dynamics much faster than observed for the standard (longitudinal) imbibition tests (cf. Fig. 1). This confirms that some specific effect damping the dynamics occurs in that case.

We now look at the water transport inside the sample, i.e., at a distance larger than $200 \mu \mathrm{m}$ from the vertical edges in contact with water. Now we systematically observe an upward motion of water, basically in the vessels (see Fig. 3), but we can also observe that fibers progressively fill upwards (see Fig. 4). However, in strong contrast with the curved shapes around trapped bubbles observed above, during such a longitudinal water imbibition the air-liquid interfaces in the vessels are almost planar (see Fig. 3). Another original observation is (a)
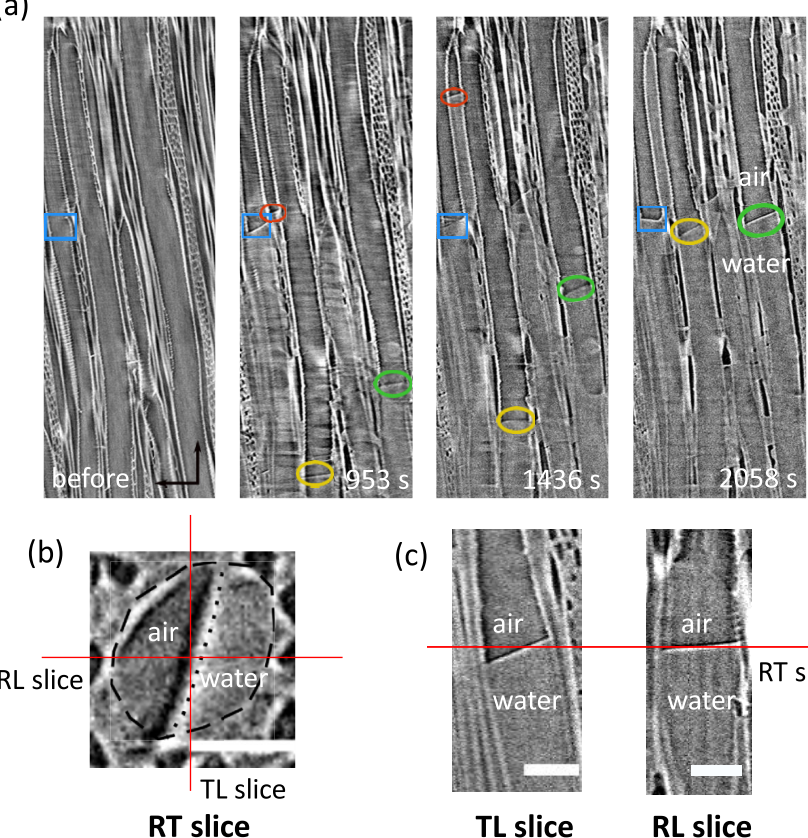

(c)

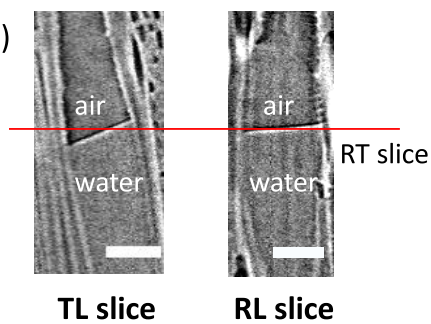

FIG. 3. Air-liquid interface dynamics and shapes (in different directions) inside vessels during longitudinal imbibition. (a) Time sequence micrographs of water imbibition of a tangential-longitudinal section of a hornbeam sample showing a few interface movements (from bottom to top). Colored circles (red, green, yellow) show different planar interfaces advancing in vessels with different velocities, while blue rectangle indicates an inclined interface stopped during the observation time by a perforation plate (visible on the first image, before interface arrival). (b) Aspect of a typical air-liquid interface formed during imbibition of hornbeam wood imaged at the transversal plane (RT slice) at the point where the radial, tangential and longitudinal planes intersect perpendicularly [see Fig. 1(a) for details]. The water/air meniscus is indicated by dotted lines and the vessel wall by dashed lines. (c) Tangential-longitudinal (TL) and radial-longitudinal (RL) cross-sectional images of a planar air-liquid interface formed in these samples. Scale bars are (a) 200 and [(b) and (c)] $50 \mu \mathrm{m}$.

that these planar interfaces do not necessarily intersect the vessel walls with an angle close to $90^{\circ}$. They may exhibit a significant apparent inclination (up to about $30^{\circ}$ ) with regards to the vessel axis (see Fig. 3). This suggests that they may be pinned to the wall by some unevenness of the wall, such as the perforation plates or the helical thickenings in the vessel elements.

From the successive images (scans at four times) in a given longitudinal section we can study the dynamics of these interfaces. For each scan, 30 TL slices, separated regularly by 50 pixels in the radial direction across the sample, are selected and used for analysis. This allows the observation of 44 interfaces in vessels during the four serial scans. It may be noticed that despite the relatively long duration of the scan (see Appendix B) the phase-contrast $\mathrm{x}$-ray imaging allowed to enhance the water-air interface distinction. Also note that this distinction of such interfaces was certainly facilitated by the fact that they advance by steps, so that each interface remains in a given position a sufficient time with regards to our measurement time to induce a sufficient contrast in the 

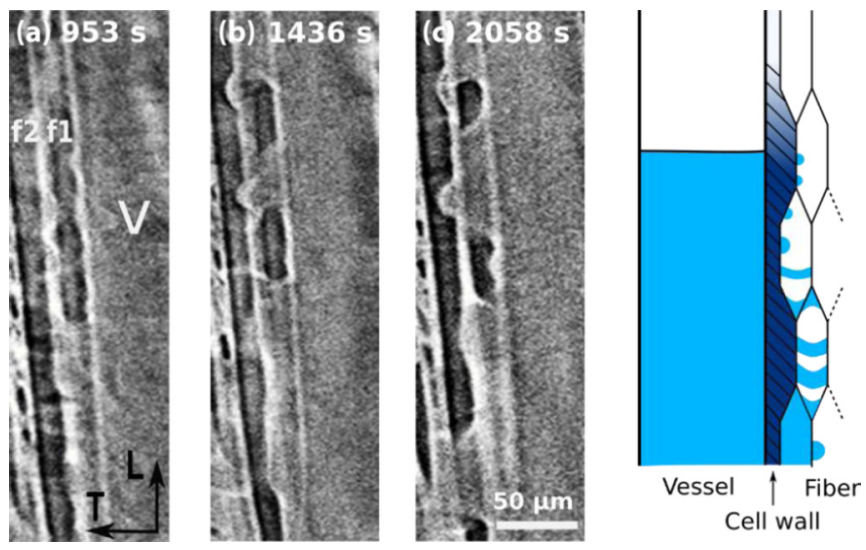

FIG. 4. (Left) Image sequence at different times during imbibition, showing how water invades the fibers around a vessel beneath the water/air interface (situated much above this region). V: vessel filled with water. f1: fiber close to vessel V, f2: fiber farther from vessel V. (a) (953 s) f1 already partially filled with water, f2 apparently free of water; (b) (1436 s) f1 goes on filling with water, first liquid interface appear in f2; (c) (2058 s) f1 and f2 go on filling. (Right) Schematic of the proposed mechanism of water penetration in wood. The liquid in the vessel can advance only when a sufficient amount of bound water has diffused in the cell walls ahead, but on average the interface keeps a planar shape. Bound water diffuses transversally and finally reaches fibers. Then due to capillary effects, free water films and blobs develop at the fiber walls, and grow in time until the corresponding free water has fully invaded the fibers.

final picture resulting from reconstruction. Following their longitudinal position in the successive images, we can compute their average velocity at each of the three steps (see Fig. 5). The order of magnitude of the average velocity, i.e., $10^{-6} \mathrm{~m} \mathrm{~s}^{-1}$, is similar to that of the velocity of ascent in the vessels deduced from macroscopic measurements after 2000 s of imbibition [cf. Fig. 1(c)]. However, we observe that their dynamics is erratic (Fig. 5). Some interfaces remain fixed over the duration of our observation, others move at an approximately constant velocity, others appear to pause during some time (i.e., they keep the same position in several images) but may then advance rapidly in the next step. This suggests that there exists an effect which controls both the wetting characteristics and the motion of the interfaces.

\section{Bound water in cell walls control air-liquid interfaces}

It may be suggested that deformations undergone by the sample during imbibition will have some contributions to this process. For example, the development of deformations in the tangential direction, where these changes are larger, take the form of a wave propagating towards the sample top (see Fig. 13), with a dynamics roughly similar to that of water imbibition. However, as appears from the micrographs presented in Fig. 3(a), this does not induce a significant change in the vessel size or structure, so that another, more subtle, effect, must be active.

Deformations of dry wood during water imbibition result from the adsorption of bound water in the cell walls, which might have an impact on wetting. This adsorption is also indirectly observed through its impact on imbibition in fibers,

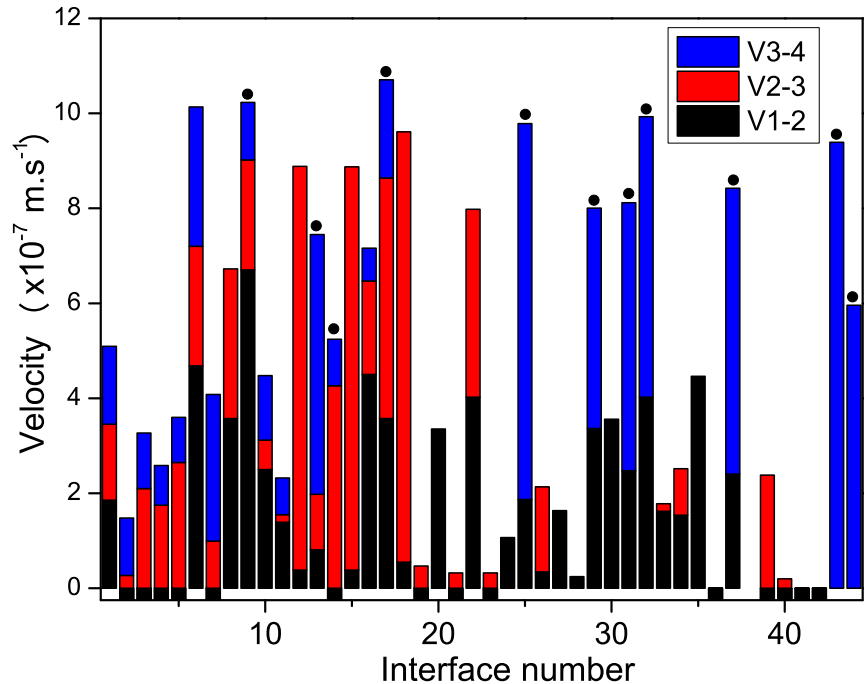

FIG. 5. Stack columns showing the distribution of apparent velocities of the 44 interfaces followed along the four scans between two successive images ( $\mathrm{V} i$ - $j$ for image $\mathrm{i}$ to image $j=i+1$, same successive times as in Fig. 4). The velocity value corresponds to the height of the corresponding colored column. A small negative value (black step) for V1-2 mentions a case for which the velocity was not measurable because the interface was out of the volume of observations in image 1 . Note that in a few cases (marked by a dot at the top) V3-4 is a lower bound of the real velocity because the interface was out of the VOI in image 4.

which are dead hollow cells closed at their ends. We can effectively observe that fiber lumens are progressively filled with water in the form of liquid water patches dispersed along their walls. The patches grow and finally gather to fill fiber lumens (see Fig. 4). Moreover, we remark that the proportion of fibers filled with water decreases when their distance from vessels increases, which confirms that water reaches the interior of fibers by moving as bound water from the vessels through the cell walls. Fibers and vessels may also be connected by pores (called pits), but free water flow through the radial nanometric pits is likely negligible within our test duration. This is supported by the fact that silicone oil, which has a smaller surface tension than water, does not adsorb to walls and has a negligible penetration into fibers during a similar period of experiment (see Fig. 6). Thus cell walls between fibers and vessels may be seen as a plain solid phase through which water can only be transported as bound water [36].

Nonetheless, bound water is also transported longitudinally in cell walls. MRI measurements specifically developed to distinguish average distribution of bound and free water in time along the longitudinal axis (see Appendix C), show that, in hornbeam, bound water advances well beyond free water, as already observed for other wood species $[17,18]$ : the bound water profiles extend significantly farther and faster than the free water profiles (see Fig. 13). Note that these profiles correspond to the average water signal over the whole sample including heterogeneous longitudinal progression of free water interfaces in the different vessels [see Fig. 1(b)], along with possible gradients of bound water in the longitudinal direction. In order to better appreciate the dynamics of bound 

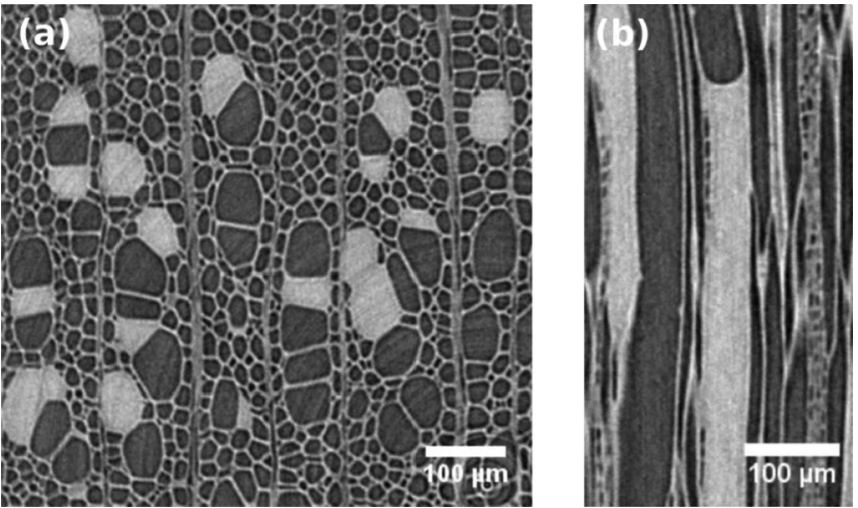

FIG. 6. Laboratory XR $\mu \mathrm{CT}$ images (see details in Appendix B) of a hornbeam sample put in contact with oil (at its bottom) during a few minutes then removed and set up for imaging. (a) RT slice of the sample. (b) TL slice showing a vessel penetrated by oil with a small contact angle $\left(30^{\circ}\right)$, surrounded by empty fibers. It appears that in contrast to water imbibition, oil penetration occurs principally in vessels. Fibers are rarely filled with oil within the duration of the scan $(18.5 \mathrm{~h}$ ), while water appears in fibers only a few minutes after the imbibition begins. Since water has a stronger surface tension suggesting that water-air interfaces should be more difficult to pass through small pores, this observation confirms a negligible role of the vessel-fiber pits in transversal water transfers. Moreover, the air-liquid interfaces in the vessels exhibit the standard expected shape associated with a low contact angle.

and free water despite this problem, we integrate the signal over the whole sample length and deduce the equivalent height of sample whose free water porosity would be fully saturated with the current volume of free water if the sample was homogeneous. In the same way we compute the equivalent height of sample whose bound water "porosity" would be fully saturated with the current additional amount of bound water (from the beginning of the test). Under these conditions we see that the average height of bound water is significantly above that of the height of free water (see Fig. 7), indicating that bound water progresses faster, along the longitudinal axis of the wood sample, than free water.

However, it may be argued that the faster bound water progression can be explained solely by vapor diffusion from the liquid-air interfaces inside the vessels, then sorption in cell walls above this interface. The impact of this effect on bound water content may be estimated by assuming a steady-state vapor diffusion process from the (quasistatic) liquid-air interface to the sample to (which leads to a uniform RH gradient), and using independent adsorption measurements (see Appendix D). Finally we see that the amount of bound water obtained under these assumptions is not negligible, but after one hour there is a significantly larger amount of bound water present in the wood which cannot result from vapor adsorption (see Fig. 7). Instead this phenomenon can be explained by the longitudinal diffusion, inside the cell walls, of bound water from the saturated regions in contact with free water towards low saturation regions.

This suggests that the presence of bound water in the cell walls would play a major role on the wetting properties. Indeed, such notion is supported by the distinct shape of

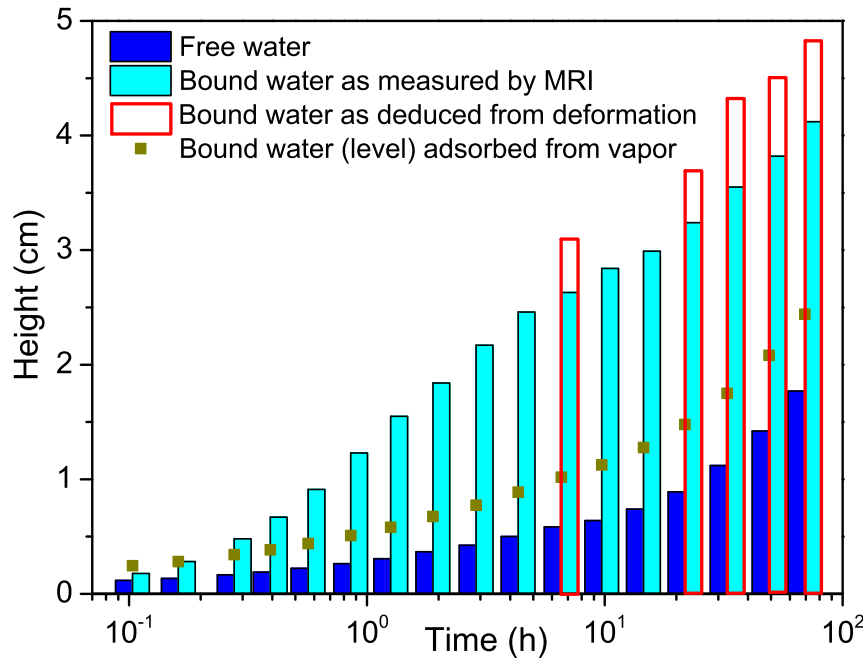

FIG. 7. Average heights of ascent of free and bound water during imbibition of hornbeam wood. The height reached by free water (dark blue) and bound water (light blue) in the sample as a function of time is estimated from MRI measurements of hornbeam wood samples. Also shown are the estimated bound water heights resulting solely from vapor adsorption (squares) and those retrieved from deformation measurements (open red) (see text).

the air-liquid interface in vessels whose surrounding fibers are partially or fully filled with water in the vicinity of the interface (i.e., cell walls are likely saturated with bound water) (see Fig. 2) and that observed in vessels where fibers above this interface are essentially empty (i.e., cell walls are likely only partially saturated with bound water) (see Fig. 3). Thus it seems that as long as the cell walls are not saturated with bound water, capillary forces are unable to induce interface displacement (contact angle close to $90^{\circ}$ or pinning), but good wetting conditions are recovered when the cell walls are saturated. This contrasts with the good wetting observed for oil [small contact angle in Fig. 6(b)] along intact cell walls, which is consistent with the macroscopic measurements (see Fig. 1).

Finally, the whole process can be qualitatively described in a relatively simple scheme: capillary uptake of water is possible only after sufficient adsorption of bound water in the cell walls; this bound water progressively invades the fibers as free water; when the cell walls are saturated with bound water capillary motion can start again but soon stops when the contact line meets a region nonsaturated with bound water. Actually this advance should be in general very slow and continuous since we expect the longitudinal profile of bound water concentration to vary continuously in time and space. However, it is likely that the material heterogeneity induces some more chaotic variations and motions of the interface, in agreement with our observations (Figs. 3 and 5). The above description also relies on a definition of the condition of bound water saturation, which is in fact critical as this is the criterion which determines the further progression of the interface. Strictly speaking we cannot have to wait for full saturation as it can be obtained only from the contact of free liquid with the cell wall. Indeed, with such a condition, the interface would never advance: the wood just above it would never reach this saturation level because each of the above processes for bound 
(a)

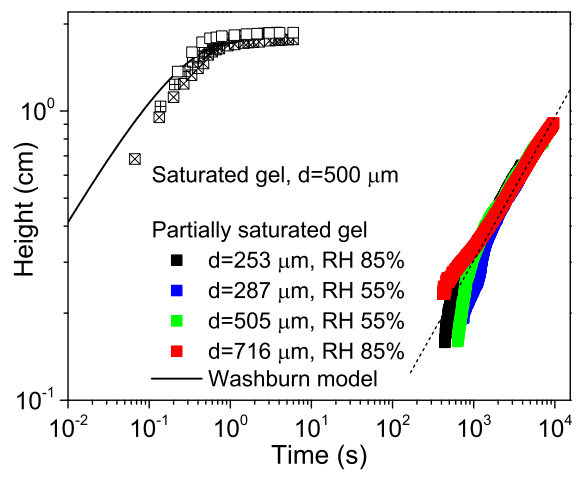

(b)

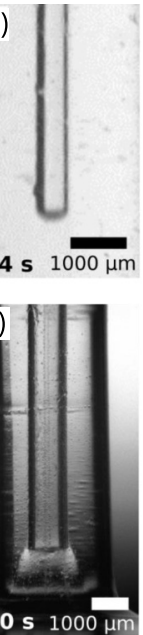

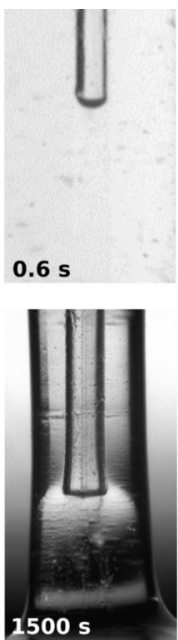
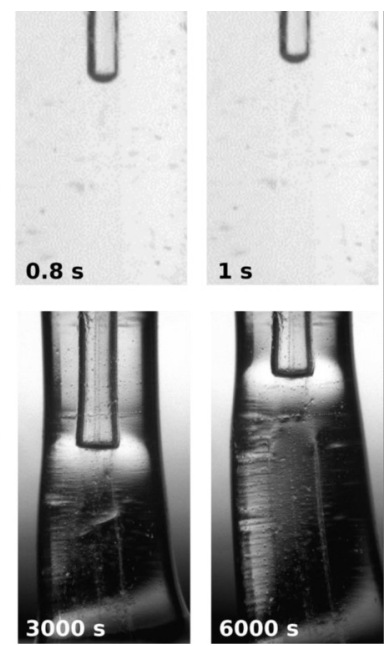

FIG. 8. (a) Water height of ascent through a templated channel inside hydrogel samples that are saturated (black and white symbols) or partially saturated (coloured symbols). Three independent measurements are shown for the saturated samples (distinct black and white symbols, channel diameter $500 \mu \mathrm{m}$ ). The continuous line corresponds to the Washburn model [Eq. (1)] fitted to these data. For partially saturated samples, the coloured symbols represent samples with distinct channel diameter $(d)$ and prepared at different relative humidity (RH): black squares, $d=253 \mu \mathrm{m}$, RH $85 \%$; blue squares, $d=287 \mu \mathrm{m}$, RH 55\%; green squares, $d=505 \mu \mathrm{m}$, RH 55\%, red squares, $d=716 \mu \mathrm{m}$, RH $85 \%$. The dashed line (of slope $\frac{1}{2}$ ) corresponds to the predicted water according to the diffusion model [Eq. (5)]. Time sequence images of water climbing through a central channel inside a hydrogel fully saturated (b) or nonsaturated (c). In the saturated case the first interface is situated at $1.5 \mathrm{~cm}$ from the sample bottom. Note that optical effects make the real diameter appear larger when looking from exterior. The channel diameters are (a) 500 and (b) $716 \mu \mathrm{m}$ and scale bars are $1000 \mu \mathrm{m}$.

water transport results from a gradient of concentration, which implies that the maximum value is reached for an infinite time. More likely there exists a critical value of saturation, slightly smaller than the full saturation, which allows a further advance of the interface. This means that the rate of advance may be somewhat difficult to predict exactly as it could significantly vary with local heterogeneities of the material inducing heterogeneities of the bound water concentration, with some unevenness of the wall surface shape affecting the capillary effects (pinning), or with some variation of the wall surface components. This explains that the planar interface may form various angles with regards to the longitudinal axis, instead of being exactly perpendicular, due to some uneven advance at different positions of the vessel walls (see Fig. 3). Moreover, this explains the erratic advance of the interfaces, which has its origin in the sudden unpinning of the interface, after some period of pinning (see Fig. 3), then displacement along the wall region which has been saturated with bound water during the blockage period due to pinning.

\section{Imbibition is also damped by bound water in hydrogels}

We can check the validity of this physical interpretation and support the generality of this phenomenon by looking at a model system - a thin channel, representing a typical hardwood vessel, inside a solid structure able to absorb water, representing the cell-wall structure. Thus, this is not a "model wood", but just a system reproducing qualitatively the geometrical and wetting or adsorption properties in and around a wood vessel. In that aim we use a polyethylene glycol (PEG)based hydrogel block, a solid cross-linked polymer network able to absorb water where a cylindrical channel has been templated from top to bottom (see Appendix E). Immersed in water for a sufficient time the hydrogel sample swells and finally reaches an equilibrium state that we associate with full (bound) water saturation. If we put the bottom of this fully saturated hollowed hydrogel piece in contact with water, the liquid rapidly climbs along the channel [Fig. 8(a)], forming a curved air-liquid interface of constant shape [Fig. 8(b)], then it stops at a critical height. This imbibition dynamics is consistent with the Washburn model [Fig. 8(a)] using $\theta=$ $73^{\circ}$. On the other hand, image analysis of the interface in the channel suggests a value around $15^{\circ}$. This discrepancy has already been described for similar (saturated) material and explained as a localized deformation of the channel wall around the contact line [37]. Note that unlike previous works here we do not consider the interplay between capillary effects and induced deformations of the structure $[37,38]$.

If we now immerse a hydrogel piece that has been previously dried and stabilized at some given RH (different from $100 \%$ ), the interface ascent is about ten thousand times slower than that measured for a saturated hydrogel, regardless of the channel size [Fig. 8(a)]. Moreover, the hydrogel swells around and beyond the interface [Fig. 8(c)]. A rounded front appears, which approximately marks the frontier between the deformed and nondeformed regions. Moreover, the air-water interface has now a planar aspect with a contact angle near $90^{\circ}$ [Fig. 8(c)]. In similarity with hardwood, even if the ascent evolves with the square root of time [Fig. 8(a)], the Washburn model does not fit to the data without a very large discrepancy on either the dynamics or the maximum height of ascent.

In this context it is reasonable to assume that the distinct wetting behaviour of saturated and nonsaturated hydrogels results from different concentrations of water absorbed in the gel. This would not be so surprising since it has been shown that water poorly wets a dry hydrophilic polymer material 

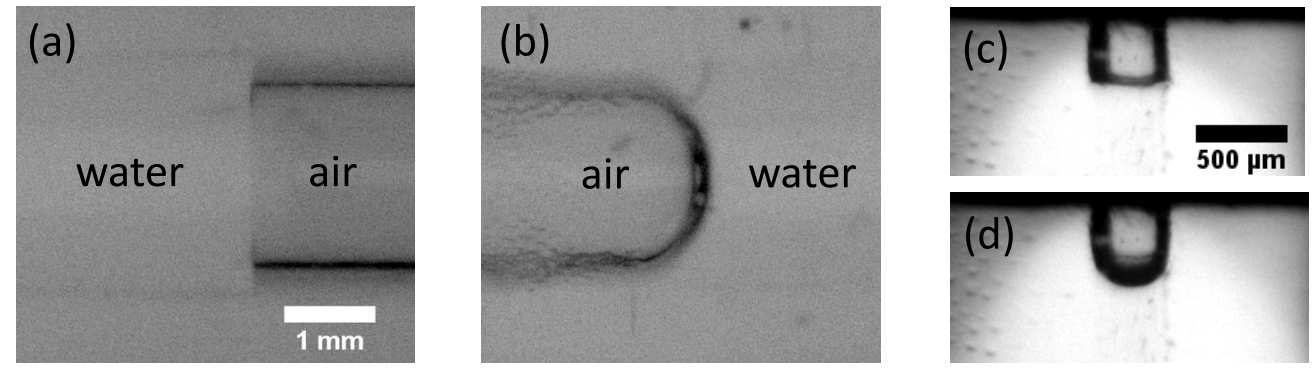

FIG. 9. (a) Air-liquid interface formed by water injection by a syringe in a 2-mm-diameter channel of a hydrogel sample pre-stored at $55 \%$ RH. Note that similar results were observed for samples stored at $85 \%$ RH. (b) Air-liquid interface formed by air injection in the same channel filled with water in (a). The direction of injection is from left to right for both (a) and (b). Aspect of the interface during spontaneous imbibition (c) and (d) immediately after a slight extraction of water from the previous situation. Note that this process leads to a minor lowering of the interface level, inducing a strong change of the interface shape.

surface, likely because a hydrophobic $-\mathrm{CH}$ structure covers this surface [39], and wetting is increased when a higher amount of water is adsorbed in a polymer layer $[40,41]$. To confirm this assumption, we inject liquid in the channel of a nonsaturated sample, at a velocity (typically about $1 \mathrm{~mm} \mathrm{~s}^{-1}$ ) much larger than spontaneous imbibition or sorption (confirmed by the absence of visible sample deformation). The observed contact angle is close to $90^{\circ}$ [Fig. 9(a)], which clearly corresponds to the wetting characteristics of a nonsaturated wall vessel. We then saturate the same sample with water and inject air inside the filled channel at the same velocity as above. In this case, the liquid withdraws with a contact angle around $15^{\circ}$ [Fig. 9(b)]. This clearly shows that the contact angle of liquid with the surface of a saturated sample strongly differs from that with a nonsaturated sample. A possible explanation is that near the saturation point, a molecular layer of water forms, covering the free surface of the sample thus resulting in good wetting. Note that if, at some time during the spontaneous imbibition, we withdraw a very small amount of water, inducing a very slight lowering of the water level in the channel, the initially rather straight interface [Fig. 9(c)] turns immediately to a rounded shape [Fig. 9(d)], as that observed during imbibition of a saturated sample [Fig. 8(b)]. This shows that the critical water saturation that leads to good wetting has been reached below the interface. We can thus now explain the slowing down of imbibition in nonsaturated gels. The motion of the air-liquid interface in the channel is negligible as long as the wall is not saturated. It only moves forward when the walls ahead become saturated with water over some short distance, which causes a change in the contact angle and allows the interface to move forward along such distance. After this, the interface becomes arrested again until the walls ahead are saturated and this dynamic cycle is repeated.

\section{Similarity with solidification front in a liquid}

Considering that water transport is governed by water sorption into the porous walls suggests at first sight, that water ascension time is uniquely determined by the dynamics of longitudinal water diffusion into the vessel walls. However, such dynamics is more complex. Since the area of contact of liquid with the wall increases continuously as the interface progresses along the channel, the boundary conditions for water sorption change continuously, which induces a coupling between the sorption and the interface displacement. Neglecting $2 \mathrm{D}$ effects (i.e., assuming a wall of small thickness), the mechanism at the origin of the liquid-gas interface progression can be described by a diffusion process between two regions from higher concentrations (region in contact with liquid water) to low concentrations (dry region), separated by the liquid-gas interface. This leads to a simple model mathematically analogous to the progressive freezing of a liquid due to heat transfer [42].

To describe the mechanism at the origin of the liquid-gas interface progression, we can restrict our attention to the longitudinal process in a thin layer of hydrogel along the wall. This allows to assume that the concentration variations along the radial direction are negligible compared to those along the channel axis. Thus the problem may be described as a diffusion process between two regions from higher concentrations (region in contact with liquid water) to low concentrations (dry hydrogel region) separated by the liquid-gas interface. We consider the one-dimensional diffusion direction along the $x$ (i.e., the channel axis), associated with a diffusion coefficient $D$. The position of the interface is noted as $X$. At the initial time $t_{0}$ the wetted height of the channel is $X_{0}=0$. For any $x>X_{0}$, the water concentration is $c_{\infty}$ (initial water content in the dry gel, $c_{\infty}<1$ ); for $x<X_{0}$, in the hydrogel layer just in contact with the liquid, the concentration is $c_{0}=1$. Then the longitudinal diffusion starts in the hydrogel, leading to an increase of the water concentration for $x>0$; when the concentration just above the interface reaches the critical value $c_{f}<$ 1 , the contact angle changes abruptly and allows some increment of capillary ascent, which places the liquid in direct contact with some new nonsaturated hydrogel surface; then the concentration in this increment newly covered by the liquid immediately turns to $c_{x}\left(0 \ll c_{f}<c_{x}<1\right)$ so that the concentration profile is discontinuous at $X$. Afterwards, the diffusion of water towards larger $x$ goes on, eventually leading the concentration to reach $c_{f}$ in higher regions, then allowing a further advance of the liquid interface $X$ and so on.

The process is in fact continuous, and corresponds to a specific problem of diffusion with moving boundaries (see Ref. [42]). Note that it is mathematically analogous to the progressive freezing of a liquid due to heat transfer: the current (frozen) "solid" region is that directly in contact with liquid in channel, the "liquid" region is the hydrogel situated 


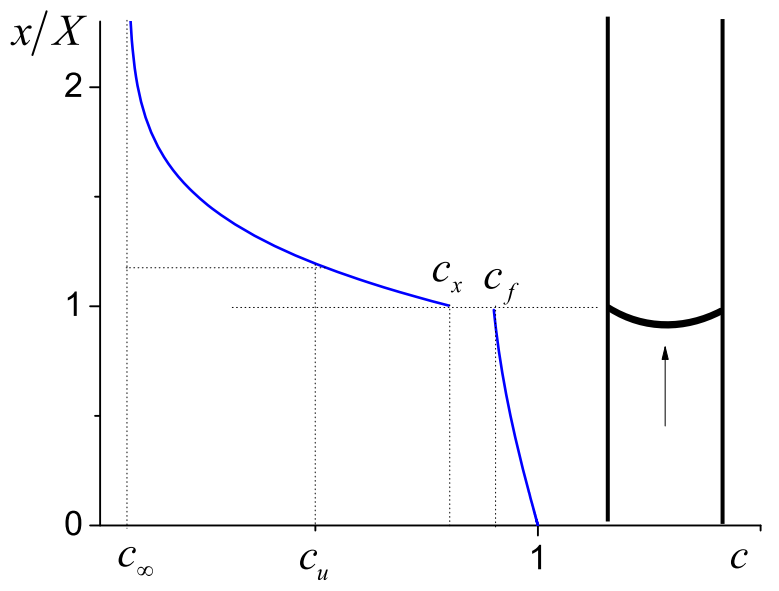

FIG. 10. Example of distribution of water concentration in hydrogel wall as a function of the dimensionless distance $x / X$ according to the model, and (left) scheme of the channel with the liquid-air interface (thick line) displacement. The liquid-gas interface is situated at $x / X=1$.

above; the concentration is equivalent to the heat content in the material; as the heat loss propagates upwards, more liquid is frozen. The solution of this problem expresses as [42]

$$
c(x, t)=c_{\infty}+\left(c_{f}-c_{\infty}\right) \frac{\operatorname{erfc}(x / 2 \sqrt{D t})}{\operatorname{erfc}(k)} \text { for } x>X
$$

and

$c(x, t)=1-\left(1-c_{x}\right) \frac{\operatorname{erf}(x / 2 \sqrt{D t})}{\operatorname{erf}(k)}$ for $0<x<X$

with $k$ a constant which depends on the values of $c_{f}, c_{x}$, and $c_{\infty}, \operatorname{erf}(z)=(2 / \sqrt{\pi}) \int_{0}^{z} \exp \left(-u^{2}\right) d u$ and $\operatorname{erfc}(z)=1-$ $\operatorname{erf}(z)$. The position of the interface in time is given as $X=$ $2 k \sqrt{D t}$. Note that in this simplified description we assumed that the diffusion coefficient $D$ is independent of water concentration and we neglected the impact of density difference, all aspects which could be taken into account in a more refined modelling. A typical aspect of the concentration distribution according to this model is shown in Fig. 10.

This model predicts the progression of the interface as a square root of time, which is consistent with our observations (see Fig. 1). It also obviously predicts the independence of the imbibition dynamics on the capillary force associated to the channel size (see Fig. 8). Moreover, this model predicts a square root variation with time of the distance at which some specific concentration is reached ahead of the interface, as this corresponds to a constant value of $x / 2 \sqrt{D t}$ in the equation for $x>X$. We can hardly check this experimentally as this would require a precise measure of the distribution of water concentration over relatively short times. However, we can follow the position of the front of deformation [top of the white region in Fig. 8(c)], which may be assumed to correspond to a constant concentration value (e.g., $c_{u}$ in Fig. 10), and see that it effectively increases with the square root of time [see Fig. 8(a)]. On the other side, we did not observe any impact of the initial concentration $c_{\infty}$ in our range of tests, whereas the diffusion is influenced by this concentration through $k$ (see above). Note that the water content at equilibrium for the hydrogel samples previously stored at $54 \%$ and $85 \% \mathrm{RH}$ is 8 and $25 \mathrm{wt} \%$, respectively, compared to $87 \mathrm{wt} \%$ when the gel is fully saturated. One possible explanation is that the critical value $c_{f}$ is very close to saturation and rather large compared to $c_{\infty}$, so that the solution of the equation for $k$ does not vary much as long as $c_{\infty}$ remains small with respect to $c_{f}$. Finally, it should be noticed that the above model does not take into account gravity effects, but we expect that at some point the imbibition curve (for the free water) will deviate from this prediction and finally stabilize at a height $x_{c}=2 \sigma \cos \theta / \rho g R$ with $\theta$ the contact angle in saturated hydrogels.

One major prediction of this model is that the liquid-air interface propagates following a square root of time law with a factor independent of the contact angle, the permeability and the viscosity, but proportional to the diffusion coefficient of bound water in the walls. This result is in strong contrast with the standard Washburn approach. With our natural systems we cannot expect to be fully predictive of the process as we can hardly, $a b$ initio, predict the exact value of the factor $(k)$, which would require a deep insight in the wetting properties of these systems, and it would also be necessary to take into account the impact of the complexity and heterogeneity of such systems.

However this model already allows to explain two fundamental aspects of our data for these systems: (i) a general square root dependence of imbibition as a function of time, even if some late regime also tends to develop with a slightly smaller exponent (say, around 0.4) [see Fig. 1(b)]; (ii) a diffusion coefficient, much lower by several orders of magnitude than expected from Washburn process, and here observed to be of the order of the typical values measured for diffusion of bound water in wood, i.e., about $D=10^{-9} \mathrm{~m}^{2} \mathrm{~s}^{-1}$ $[10,14,43,44]$, a value which is of the same order as the selfdiffusion coefficient of confined water [45]. More precisely, for the hydrogel, using the same diffusion coefficient, we find (experimentally) that the height vs time curve follows a model

$$
h=2 k \sqrt{D t}
$$

with $k \approx 1.5$ [see dotted line in Fig. 8(a)], in good agreement with the above theory. For wood the comparison of theory with (macroscopic) imbibition data is slightly more complex since we should now describe the dynamics of the total amount of water, including free and bound water. As a first approximation, assuming that the dynamics of bound water uptake is finally roughly similar to that of free water, we can expect a law of the type

$$
h^{*}=2 \varepsilon(1+\alpha) k \sqrt{D t}
$$

for the apparent height of water having penetrated the sample, in which $\alpha \approx 0.25$ is the fraction of bound water with regards to the free water amount (estimated from NMR relaxation time distribution). Here this model fits the data for hornbeam with $k \approx 3$ [see dotted line in Fig. 1(c)].

To sum up, this "diffusion" model predicts that the airliquid interface propagates as a square root of time law with a factor independent of the contact angle, the permeability (channel diameter) and the viscosity, but proportional to the diffusion coefficient of bound water in the walls, which 
strongly contrasts with a capillarity-driven process. Finally, the resulting diffusion coefficient (for imbibition) can be much lower by several orders of magnitude than expected from Washburn process (i.e., $K$ ), in agreement with our experimental data [Figs. 1(c) and 8(a)].

\section{CONCLUSION}

The fact that the motion of liquid in vessels through wood is controlled by bound water adsorption in cell walls means that if some empty wood vessel is placed in contact with water, it will let it penetrate farther only when its surrounding structure has been filled with bound water. The relevance of the process for plant materials, able to store bound water, was demonstrated with a model material. This process might contribute to a regulation of water absorption for these materials after some drying period, allowing them to first progressively store available water as bound water, instead of leaving it rapidly flow through the structure and move out for example by evaporation. This kind of interplay between bound and liquid water might also play some role in the recovery of plants from cavitation. Finally, it also might aid in the development of bio-inspired porous materials able to absorb liquid with a tunable timing. The exact physical origin of the strong contact angle change with the amount of adsorbed bound water in the walls is a physicochemical mechanism which remains to be explained. We can suggest that it comes from the adsorption of the extremely thin liquid film close to the line of contact (when the wall is not saturated), which scales as the square root of time, which can be faster than the expected interface displacement due to capillary effects.

\section{ACKNOWLEDGMENTS}

This work has benefited from a French government grant managed by ANR within the frame of the national program "Investments for the Future" ANR-11-LABX-022-01. We also acknowledge fruitful discussions with Patrick Huber and Olivier Pitois.

M.Z., S.C., M.B., A.K., P.A., and M.B. carried out the Synchrotron experiments and analyzed these data. M.Z., S.R., and D.C-M. carried out the MRI experiments and analyzed these data. M.Z. and G.G. fabricated the hydrogel materials. M.Z., S.C., S.R., and P.C. interpreted the results. The authors declare no competing interests.

\section{APPENDIX A: HARDWOOD STRUCTURE}

The structure of hardwood can be considered basically made of very long parallel vessels (typical radius from several tens to several hundreds of microns) in a matrix containing elongated closed fibers (with a radius around a few microns) of limited length and cell walls (see Ref. [3]). A vessel is in fact composed by a series of individual cells, whose end walls are partially or completely dissolved during the period of cell maturation, thus leaving the structure called perforation plates (simple or scalariform) between each unit and forming a long capillary [3]. Vessels may appear as clusters: two vessels share a cell wall along which small openings (intervessel pits) are distributed allowing some communication between them. Fibers have a reduced number of pits to allow direct flow between vessels and fibers (simple or bordered pits, etc.). There are also some independent cells oriented in the radial direction (ray cells) and representing about 5\%-10\% of the wood volume; in principle they play a negligible role within the frame of our study of the liquid transport in the longitudinal direction. Tracheids may be present in some species, but their contribution in the water transport may be considered as negligible.

The different wood types we tested allow to cover the different types of hardwoods, i.e., including semidiffuse, diffuse-porous, and ring-porous hardwoods. The size and the distribution of the vessels in diffuse-porous species are fairly homogenous across a growth-ring while in ring-porous ones, vessels are much larger and numerous in earlywood than in latewood. Estimations of their geometrical characteristics are provided in Table I. Imbibition tests as shown in Fig. 1 are performed on the four species but only hornbeam was analyzed through x-ray microcomputed tomography imaging and Magnetic Resonance Imaging. In this case, the vessels are distributed fairly evenly across the earlywood (EW) and latewood (LW). For all the experiments, wood materials were collected from planks which had been left drying naturally under ambient conditions, the samples were cut along the anisotropic directions of wood: the longitudinal (L) direction (vertical axis of the tree), which is parallel to the imbibition axis, and the radial $(\mathrm{R})$ and tangential $(\mathrm{T})$ directions. They were then stored at $54 \% \mathrm{RH}$ before experiments.

\section{APPENDIX B: X-RAY MICROCOMPUTED TOMOGRAPHY IMAGING}

$\mathrm{XR} \mu \mathrm{CT}$ consists in recording many $\mathrm{x}$-ray absorption radiographies of a sample taken from different viewpoints, usually obtained by rotating the sample. A reconstruction algorithm produces a 3D image of the sample, discretized in cubic voxels with a size depending on the geometry of the setup. The grey levels of the voxels essentially depend on the density and the atomic number of the sample constituents.

Synchrotron $\mathrm{XR} \mu \mathrm{CT}$ was carried out at the Psiché beamline of the French national synchrotron facility Soleil [46] which allows phase-contrast x-ray imaging. 1000 projections equally distributed over a rotation angle of $180^{\circ}$ were acquired. Monochromatic beam illumination was used with an energy of $24.9 \mathrm{keV}$. The detector system uses a $90-\mu$ m-thick LuAG scintillator to convert $\mathrm{x}$ rays to a visible light image. This image is relayed to a Hamamatsu ORCA Flash4.0 sCMOS camera using a Mitutoyo 5x microscope objective, giving an effective pixel size of $1.3 \mu \mathrm{m}$. Volumes were reconstructed with the software pyHST2 [47] using Paganin filter and their dimensions were $2048 \times 2048 \times 1800$ voxels $^{3}$. For the Synchrotron XR $\mu$ CT one reference image of the sample before water imbibition and 4 serial scans were obtained for the in situ analysis. The measurement time was slightly less than $7 \mathrm{~min}$ and the interval between each scan beginning was $8 \mathrm{~min}$. 
TABLE I. Estimates of the geometrical characteristics of the tested specimens.

\begin{tabular}{|c|c|c|c|c|c|}
\hline & Type & Vessel diameter $(\mu \mathrm{m})$ & Vessels per $\mathrm{mm}^{2}$ & Fibers diameter $(\mu \mathrm{m})$ & Fibers, tracheids, etc. per $\mathrm{mm}^{2}$ \\
\hline Hornbeam & Diffuse-porous & 60 & 110 & $4-30$ & $2000-2100$ \\
\hline Poplar & Semidiffuse-porous & 110 & 50 & $5-40$ & $1300-1400$ \\
\hline Oak & Ring-porous & $\begin{array}{c}200-250 \text { EW } \\
40-60 \mathrm{LW}\end{array}$ & $\begin{array}{c}1-2 \mathrm{EW} \\
20-50 \mathrm{LW}\end{array}$ & $10-20$ & 3800 \\
\hline Ash & Ring-porous & $\begin{array}{c}200-250 \mathrm{EW} \\
20-80 \mathrm{LW}\end{array}$ & $\begin{array}{c}3-8 \text { EW } \\
20-30 \text { LW }\end{array}$ & $5-20$ & $2000-2500$ \\
\hline
\end{tabular}

The sample size was $1.75(\mathrm{R}) \times 1.75(\mathrm{~T}) \times 6(\mathrm{~L}) \mathrm{mm}^{3}$. To reduce the volumes of data treated, observations were not made on the whole height of the specimen, but instead a volume of interest (VOI) with dimensions $1600(\mathrm{R}) \times 1500(\mathrm{~T}) \times$ 1024(L) voxels was used. Figure 11 shows a reconstructed 3D image for a smaller volume $(300 \times 300 \times 300$ voxels $)$ inside the sample.

Besides, images for oil imbibition were acquired with a conical beam laboratory RX-Solutions x-ray microtomograph (model Ultratom). It was equipped with a Hamamatsu $160 \mathrm{kV}$ $\mathrm{x}$-ray tube (model L10711-23) and a Photonic Science sensor (model VHR). The intensity and voltage in the $\mathrm{x}$-ray tube were respectively $100 \mu \mathrm{A}$ and $70 \mathrm{kV}$. Every image was the result of averaging 6 frames, which individual exposure time was $0.25 \mathrm{~s}$. Voxel size was set at $1.7 \mu \mathrm{m}$. 1536 projections were recorded during a helicoidal scan. Slices were reconstructed using $\mathrm{x}$-act reconstruction software, and their dimensions were $1186 \times 1518$ pixels. The procedure for observing oil imbibition in hornbeam is as follows: the sample bottom is put in contact with an oil bath during $2 \mathrm{~min}$, so that the average imbibition height is around the mi-height of sample, then it is removed from the oil bath and placed in the x-ray tomograph for imaging (see above). The oil used here is the silicone oil 47V350, obtained from Chimie Plus (viscosity and surface tension at $25^{\circ} \mathrm{C}: 0.35 \mathrm{~Pa} \mathrm{~s}, 0.0210 \mathrm{~m}^{-1}$ ). The absence of image blurring proves that the oil negligibly moves in the sample during this period. For the laboratory $\mathrm{XR} \mu \mathrm{CT}$ test with oil the measurement times was 18.5 hours to allow a full scan of the 17 high sample to be measured. The sample size was about $2(\mathrm{R}) \times 2(\mathrm{~T}) \times 17(\mathrm{~L}) \mathrm{mm}^{3}$ for experiments with oil.
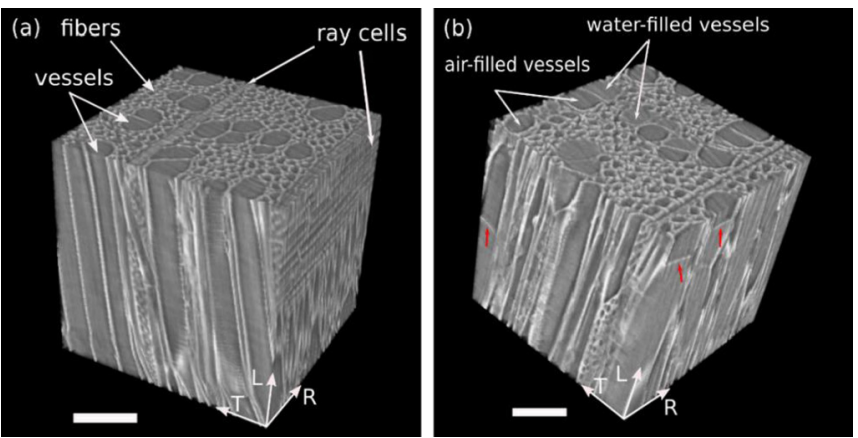

FIG. 11. 3D rendering of synchrotron XR $\mu \mathrm{CT}$ images. (a) Cellular structure of a hornbeam sample before imbibition. (b) Hornbeam sample imbibed with water. Water-filled vessels show lighter graylevels than those without water. Some planar water-air interfaces in vessels are visible and indicated by red arrows. Scale bars, $100 \mu \mathrm{m}$.
APPENDIX C: MAGNETIC RESONANCE IMAGING

\section{Setup}

The distribution of liquid content along the sample axis (see Fig. 12) was measured by a vertical MRI spectrometer (DBX 24/80 Bruker) operating at $0.5 \mathrm{~T}(20-\mathrm{MHz}$ proton frequency) and equipped with a ${ }^{1} \mathrm{H}$ birdcage radio frequency coil of $20 \mathrm{~cm}$ inner diameter. In our working conditions, the measurements are principally sensitive to water molecules contained in the sample. The water content was studied as either projected profiles along the vertical axis, or 2D pictures in a vertical slice.

\section{1D Imaging sequence}

The NMR profiling sequence utilized in this study was a 1D spin-echo sequence using a space encoding gradient in the vertical direction, where the echo is actually repeated 16 times as a series following $\pi / 2-\operatorname{grad}-(\pi-\operatorname{grad})_{16}$ with a regular inter-echo delay TE. The NMR signal owing to each echo was recorded by means of a 32 steps duplex cogwheel phase cycling scheme [48]. For each measurement, a time series of NMR profiles was then obtained for a series of recording times TE, ...,16TE. The experiment was performed

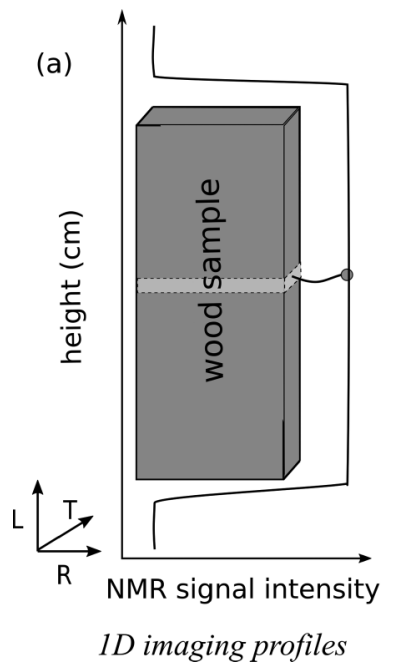

(b)

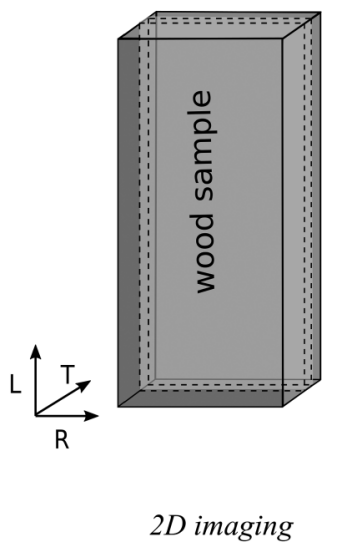

FIG. 12. (a) Projection of transverse signal for $1 \mathrm{D}$ profiles along sample axis and (b) slice selection for 2D NMR imaging. For 1D profiles, every point corresponds to the integrated signal in the horizontal slice (showed as the light gray cuboid) with $1.25 \mathrm{~mm}$ of thickness. The 2D imaging is realized as a vertical slice with $2 \mathrm{~mm}$ of thickness (in the tangential direction) parallel to the RL plane. 
at the magnetic center of our gradient coil (a BGA26 by Bruker, $26 \mathrm{~cm}$ inner diameter) with a field of view of $16 \mathrm{~cm}$. The resolution along the vertical axis was $1.25 \mathrm{~mm}$. Slight profile distortion due to possible gradient nonlinearity was observed. In order to eliminate this, all the profiles were normalized by a referential profile of a cylinder glass tube with known dimensions containing the same liquid. Our measurements were not subjected to any spin-lattice NMR relaxation $\left(T_{1}\right)$ weighting, because the recycling delay between scans was set to 5 times of the longest $T_{1}$ of water found in our sample. The gradient strength was about $0.035 \mathrm{~T} \mathrm{~m}^{-1}$ with $500 \mu$ s stabilizing time and freshly tuned pre-emphasis which removed eddy effects at the end of this stabilizing time. The echo time was $\mathrm{TE}=3.47 \mathrm{~ms}$. A Gaussian filtering was used to reduce spurious Gibbs oscillations due to the high signal contrast at the interface between the water reservoir and the bottom of the sample.

The successive echoes permitted the local analysis of spinspin $\left(\mathrm{T}_{2}\right)$ relaxation times of water at short times. A separate study of the full $\mathrm{T}_{2}$ distribution of the sample containing bound and free water showed that three water pools can actually be identified, exhibiting three different relaxation times. The fast relaxing component was attributed to bound water, whereas the two slower components were attributed to possibly two types of free water. However, we are still unable to understand the origin of these two populations. It would be natural to consider that they correspond to the two types of structural elements according to pore sizes: the medium peak would correspond to free water in fibers while the other one to free water in vessels. However, the ratio of the intensity of the two peaks did not correspond to the volume ratio of fibers and vessels as measured by image analysis of microscopic observations, so that we could not definitely conclude on that point. As a consequence, we thought it preferable to speak of free water as a whole and avoid trying to distinguish two populations without clear physical interpretations.

At the time scale of the NMR sequence, the two pools of free water actually appeared as one single pool with slow relaxation, so that a biexponential analysis of the signal intensity was used, following that previously introduced in Ref. [49]. MRI data were regarded as if each layer of our sample would contain two water pools with local relaxation $T_{2, \mathrm{~b}}(z)$ and $T_{2, \mathrm{f}}(z)$ (corresponding to relaxation of bound and free water seen as a whole). Denoting $m_{0, \mathrm{~b}}(z)$ and $m_{0, \mathrm{f}}(z)$ the actual spin density profile of bound and free water, respectively, the profiles obtained from each $n$ echo were then modelled as

$$
\begin{aligned}
\operatorname{prof}_{n}(z, n)= & m_{0, b}(z) \exp \left(-\frac{n T E}{T_{2, b}(z)}\right) \\
& +m_{0, f}(z) \exp \left(-\frac{n T E}{T_{2, f}(z)}\right) .
\end{aligned}
$$

On some numerical tests, it was checked that the use of a biexponential instead of a triexponential analysis was of little quantitative influence on the determination of the total amount of free water, and also greatly improved the stability of the fitting procedure.

The quantification of bound and free water pools was expected to be correct at high RH \%. Bound water indeed has a $T_{2}$ value close to $4 \mathrm{~ms}$, which makes it correctly detectable

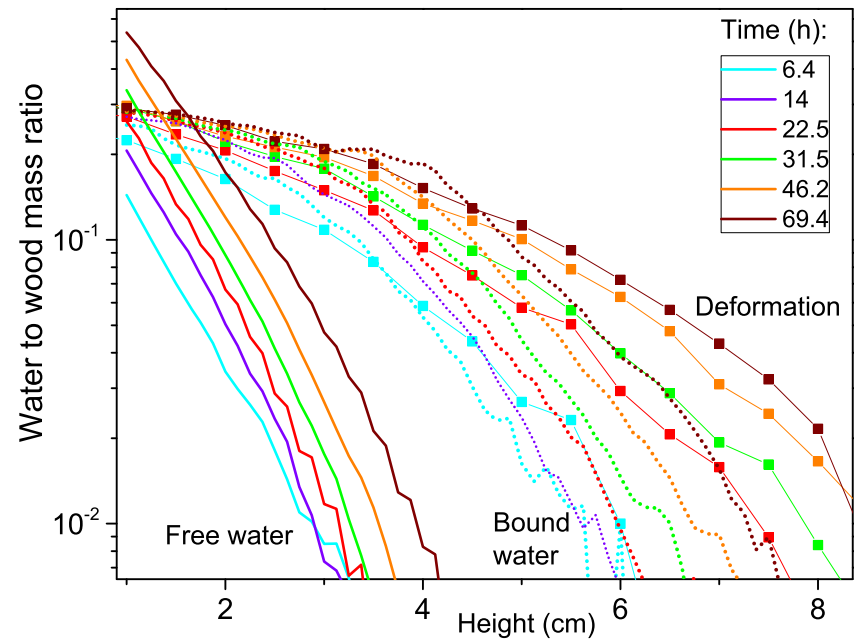

FIG. 13. Aspect of some of the concentration profiles obtained for free water (continuous lines), bound water (dotted lines) through MRI, and the equivalent amount of water associated with the sample deformation (symbols + continuous lines).

by the NMR sequence. At low RH \% however, this $T_{2}$ value can get shorter than $1 \mathrm{~ms}$, and lead to underestimation. As far as data interpretation is concerned, in the regions of lower bound water content, and even if the NMR signal is no longer proportional to the bound water content, we was seen that it is still possible to appreciate quantitatively the dynamics of bound water from the evolution of the shape of the NMR signal distribution along the sample height.

\section{3. $2 D$ imaging}

Five simultaneous 2D MRI vertical slices of 2-mm thick with 2-mm interval between them (parallel to RL plane, see Fig. 12) passing through the wood sample with space resolution equal to 0.47 (radial) $\times 2.19$ (longitudinal) $\mathrm{mm}$, were taken at different times during wood imbibition. A multi-spin multi-echo (MSME) pulse sequence acquiring 8 echoes was used with an echo time of $10 \mathrm{~ms}$ and a recycle delay of $600 \mathrm{~ms}$. A short recycle delay value and multiecho addition were chosen so as to obtain an acceptable signal-to-noise ratio and to keep measurement time under 30 minutes. It however induced both $T_{1}$ and $T_{2}$ weighting, thus preventing here direct quantification. Moreover, they did not show bound water as its $T_{2}$ value is of the order of $4 \mathrm{~ms}$. 2D images were thus essential to appreciate the distribution of liquid in the transversal direction but could only be qualitatively compared with 1D profiles of free water.

The distribution profiles for free and bound water at different times along the hornbeam sample longitudinal axis (see Fig. 13) were measured with the help of MRI. Note that the bound water profiles cannot readily be quantitatively interpreted in terms of the total bound water amount stored at some position from the beginning of the test. Indeed, the bound water being initially present in the sample (at 54\% RH) does not provide a visible NMR signal with our sequence as its relaxation time is too small. This is only when sufficient water is added in the cell walls that all the bound water (i.e., initial + added) present may become visible. More precisely, beyond 
that critical value of bound water amount, the NMR signal increases first much faster than the concentration increase, and then increases more or less proportionally to it. This effect nevertheless does not affect our analysis of the dynamics of bound water since, for our sample initially at $54 \% \mathrm{RH}$, we can consider as a first approximation that a signal appears just when new bound water has been formed, and then the signal increases with the bound water amount. Under such conditions the recorded amount of bound water should represent a lower bound of the effective additional bound water amount formed in the structure.

We can see that both profiles (bound and free water) exhibit gradients, i.e., the front of the profile is not vertical as for a progressive full imbibition process, and this is due to the heterogeneity of the material: depending on the detailed characteristics of the wood, the liquid progresses at different speeds in the different vessels [see Fig. 1(b)]. Despite this effect, we clearly see that the bound water progresses more rapidly upward than the liquid water: for each time step, the front of bound water is far ahead of the corresponding front of liquid water.

This observation is confirmed by measurements of volumetric deformation at each height at these different times with an outside micrometer Mitsutoyo with $0.01 \mathrm{~mm}$ precision in the transversal plane every $5 \mathrm{~mm}$ along the sample axis (only radial and tangential deformations are measured; longitudinal deformations are considered negligible). It is expected that these deformations find their origin in the bound water adsorbed in the solid structure. We can thus interpret the corresponding volume gain as an amount of bound water newly adsorbed. Doing that we see that the deformation profiles are rather close to the bound water profiles (see Fig. 13) (in fact they are just above, in agreement with the fact that the bound water profiles are underestimations of the effective bound water amount).

\section{APPENDIX D: IMPACT OF WATER SORPTION IN WALLS}

Since diffusion in wood can occur either as bound water or as vapor [1] there are two possible origins for the increase of bound water concentration in the region situated beyond the water-air interface in the vessel: (1) the bound water resulting from transversal adsorption tends to diffuse longitudinally upwards towards lower concentration regions, (2) bound water is adsorbed from vapor in the air, whose concentration is large around the liquid-air interface. In order to discuss these effects we can observe the dynamics of bound water and its correlation with that of the free water, from MRI measurements (see above).

Let us now examine the possible contribution of vapor adsorption to this bound water dynamics. Due to evaporation, vapor diffuses from each liquid-air interface (above which the relative humidity is $R H_{m}=100 \%$ ) to the top sample exit, where the relative humidity is $R H_{0}$. Assuming steady state the diffusion equation predicts a linear vapor gradient between these two points situated at a distance $L$. As a consequence, the vertical vessel wall situated at the level $x$ above the liquidair interface will be in contact with an air at a relative humidity $R H(x)=R H_{0}+\left(R H_{m}-R H_{0}\right)(1-x / L)$. Besides, from independent adsorption tests with $2(\mathrm{R}) \times 2(\mathrm{~T}) \times 0.5(\mathrm{~L}) \mathrm{cm}^{3}$

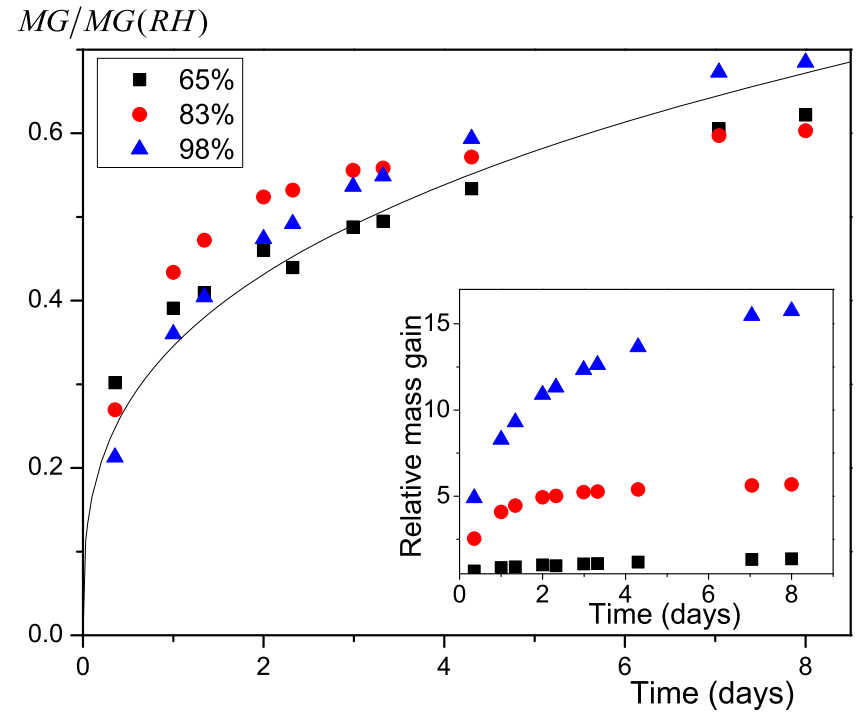

FIG. 14. Adsorption curves for small cubic samples (initially in stable state at $54 \% \mathrm{RH}$ ) placed in different $\mathrm{RH}$ (see displayed caption): ratio of the moisture gain to the maximum expected gain as a function of time (see text). The continuous line is the equation $f(t)=0.0135 t^{0.32}$ with $t$ in hours. The inset shows the $M G$ values in the range $54 \%-98 \%$.

samples (see Adsorption measurements below), it appears that in presence of air at a fixed $R H$, the wood moisture content variation can be well represented by the simple equation $M C(x, t)=M C_{0}+f(t)\left(M C(R H(x))-M C_{0}\right)$ in which $M C_{0}$ is the initial moisture content, $M C(R H)$ the equilibrium moisture content at $R H$, and $f(t)$ is a function independent of the moisture content.

We can then a priori compute the amount of bound water which would be adsorbed over a given time in the cell walls in the absence of any other effect. With this aim we integrate the adsorbed moisture content over the sample length above the interface, and we assume that the sample is immediately saturated with bound water along the region saturated with free water.

The adsorption measurements were carried out by first placing small cubic $2(\mathrm{R}) \times 2(\mathrm{~T}) \times 0.5(\mathrm{~L}) \mathrm{cm}^{3}$ samples for several weeks in a $54 \% \mathrm{RH}$, so that the moisture content is $M C_{0}$ (and the mass is $M_{0}$ ). After that they were placed in a (new) RH of either $65 \%, 83 \%$, or $98 \%$ and their mass was followed in time. Experiments were carried out at room temperature (around $20^{\circ} \mathrm{C}$ ). We can then compute the relative mass gain, i.e., the relative difference of mass between the sample in its current state and it initial mass when it was just placed in the new RH. Here we will call this relative mass gain the moisture gain $(M G)$. It is related to the moisture content via: $M G=\left(M C-M C_{0}\right)\left(M_{d} / M_{0}\right)$, in which $M_{d}$ is the dry mass.

The maximum gains $M G(R H)$ were here estimated roughly from extrapolation at long times. The corresponding values appear to be well represented by the function $0.012(R H-54)^{2}$. Remark that the exact values do not play a significant role in our approach. Indeed, the critical point within the frame of our calculation of the adsorbed vapor during our imbibition tests (see text), is that the representation 
of the adsorption be relevant within the duration of these imbibition tests (a few days only), which is the case as soon as a proper function is fitted to the data in this range. Finally we find that $M G / M G(R H)$ may be represented by the function $f(t)$ (see Fig. 14), so that we get

$$
M C(x, t) \approx M C_{0}+f(t)\left(M C(R H(x))-M C_{0}\right) .
$$

\section{APPENDIX E: HYDROGEL PREPARATION}

The PEG-based hydrogel was prepared from a solution of cross-linkers [poly(ethylene glycol) methyl ether methacrylate (average Mn 500) and poly(ethylene glycol) diacrylate (average $\mathrm{Mn} 700)$ in deionized water (72 wt\%)].

The polymer solution was gently mixed and then deaerated under vacuum for approximately $30 \mathrm{~min}$ to avoid trapped air bubbles. An aqueous solution containing $5 \mathrm{wt} \%$ ammonium persulfate as radical initiator (1 wt $\%$ to the polymer solution) and an aqueous solution with $5 \mathrm{wt} \% \mathrm{~N}, \mathrm{~N}, \mathrm{~N}^{\prime}, \mathrm{N}^{\prime}$ tetramethylethylenediamine as catalyzer ( $1 \mathrm{wt} \%$ to the polymer solution) were added to the deaerated solution. The hydrogel would be formed in several minutes. In order to form straight capillaries inside hydrogels, the solution was immediately suctioned into $1-\mathrm{mL}$ syringes with a $400-\mu \mathrm{m}$ diameter nylon string inside to form a circular cross-sectional channel. After standing $2 \mathrm{~h}$ at room temperature, the gel was ready to be removed from syringes and rinsed with de-ionized water at least three times to remove unreacted chemical compounds. The nylon string was carefully discarded leaving an empty channel inside the gel. The hydrogels were stored in de-ionized water no less than $12 \mathrm{~h}$ before being conditioned at $54 \%$ RH.
[1] W. G. Hopkins, Introduction to Plant Physiology (Wiley, New York, 1999).

[2] M. T. Tyree, The ascent of water, Nature (London) 423, 923 (2003).

[3] M. T. Tyree and M. H. Zimmermann, Xylem Structure and the Ascent of Sap (Springer, Berlin, 2002).

[4] T. D. Wheeler and A. D. Stroock, The transpiration of water at negative pressures in a synthetic tree, Nature 455, 208 (2008).

[5] O. Vincent, P. Marmottant, P. A. Qinto-Su, and C. D. Ohl, Birth and Growth of Cavitation Bubbles within Water Under Tension Confined in a Simple Synthetic Tree, Phys. Rev. Lett. 108, 184502 (2012).

[6] D. S. Guillermina, M. E. Fernandez, A. S. Sergent, P. Rozenberg, E. Badel, and A. Martinez-Meier, Dynamics of cavitation in a Douglas-fir tree-ring: transition-wood, the lord of the ring, J. Plant Hydraulics 1, e005 (2014).

[7] P. Ball, Watching wood dry, Nat. Mater. 13, 922 (2014).

[8] J. H. Van Houts, S. Q. Wang, H. P. Shi, A. J. Pan, and G. W. KabalKa, Moisture movement and thickness swelling in oriented strandboard, part 1. Analysis using nuclear magnetic resonance microimaging, Wood Sci. Technol. 38, 617 (2004).

[9] C. Silva, J. M. Branco, A. Camões, and P. B. Lourenço, Dimensional variation of three softwood due to hygroscopic behavior, Constr. Build Mater. 59, 25 (2014).

[10] J. Siau, Transport Process in Wood (Springer-Verlag, New York, 1984).

[11] G. Bramhall, The validity of Darcy's law in the axial penetration of wood, Wood Sci. Technol. 5, 121 (1971).

[12] A. J. Bolton, A reexamination of some deviations from Darcy's Law in coniferous wood, Wood Sci. Technol. 22, 311 (1988).

[13] A. C. Y. Choo, T. M. Paridah, A. Karimi, E. S. Bakar, K. Abdan, A. Ibrahim, and F. A. B. Balkis, Study on the longitudinal permeability of oil palm wood, Ind. Eng. Chem. Res. 52, 9405 (2013).

[14] K. Krabbenhoft, Moisture transport in wood - a study of physical-mathematical models and their numerical implementation, Ph.D. thesis, Technical University of Denmark, 2003.

[15] J. Ryu, B. G. Hwang, and S. J. Lee, In vivo dynamic analysis of water refilling in embolized xylem vessels of intact Zea mays leaves, Ann. Botany 118, 1033 (2016).
[16] B. G. Hwang, J. Ryu, and S. J. Lee, Vulenrability of protoxylem and metaxylem vessels to embolisms and radia refilling in a vascular bundle of maise leaves, Frontiers Plant Sci. 7, 941 (2016).

[17] Ö. Gezici-Koç, S. J. F. Erich, H. P. Huinink, L. G. J. van der Ven, and O. C. G. Adan, Bound and free water distribution in wood during water uptake and drying as measured by 1D magnetic resonance imaging, Cellulose 24, 535 (2017).

[18] M. Zhou, S. Caré, D. Courtier-Murias, P. Faure, S. Rodts, and P. Coussot, Magnetic resonance imaging evidences of the impact of water sorption on hardwood capillary imbibition dynamics, Wood Sci. Technol. 52, 929 (2018).

[19] G. Almeida, S. Leclerc, and P. Perré, NMR imaging of fluid pathways during drainage of softwood in a pressure membrane chamber, Int. J. Multiphase Flow 34, 312 (2008).

[20] P. M. Kekkonen, A. Ylisassi, and V. V. Telkki, Absorption of water in thermally modified pine wood as studied by nuclear magnetic resonance, J. Phys. Chem. C 118, 2146 (2014).

[21] B. Mazela, J. Kowalczuk, I. Ratajczak, and K. Szentner, Moisture content (MC) and multinuclear magnetic resonance imaging (MRI) study of water absorption effect on wood treated with aminofunctional silane, Eur. J. Wood Wood Prod. 72, 243 (2014).

[22] M. Sedighi-Gilani, M. Griffa, D. Mannes, E. Lehmann, J. Carmeliet, and D. Derome, Visualization and quantification of liquid water transport in softwood by means of neutron radiography. Int. J. Heat Mass Transfer 55, 6211 (2012).

[23] G. Desmarais, M. S. Gilani, P. Vontobel, J. Carmeliet, and D. Derome, Transport of polar and nonpolar liquids in softwood imaged by neutron radiography, Transport Porous Media 113 383 (2016).

[24] E. W. Washburn, The dynamics of capillary flow, Phys. Rev. 17, 273 (1921)

[25] S. Gruener, Z. Sadjadi, H. E. Hermes, A. V. Kityk, K. Knorr, S. U. Egelhaaf, H. Rieger, and P. Huber, Anomalous front broadening during spontaneous imbibition in a matrix with elongated pores, Proc. Natl. Acad. Sci. USA 109, 10245 (2012).

[26] I. Kucerova, Methods to measure the penetration of consolidant solutions into "dry" wood, J. Cultural Heritage 13, S191 (2012). 
[27] P. G. de Gennes, F. Brochart-Wyart, and D. Quéré, Capillary and Wetting Phenomena (Springer, Berlin, 2003).

[28] J. G. Salin, Modelling water absorption in wood, Wood Mater. Sci. Eng. 3, 102 (2008).

[29] J. Johansson and J. G. Salin, Application of percolation modelling on end-grain water absorption in aspen (Populu tremula L.), Wood Mater. Sci. Eng. 6, 112 (2011).

[30] T. Lerouge, O. Pitois, D. Grande, B. Le Droumaguet, and P. Coussot, Synergetic actions of mixed small and large pores for capillary absorption through biporous polymeric materials, Soft Matter, 14, 8137 (2018)

[31] J. Ha, J. Kim, Y. Jung, G. Yun, D. N. Kim, and H. Y. Kim, Poro-elasto-capillary wicking of cellulose sponges, Science Advances 4, eaao7051 (2018).

[32] J. Kim, J. Ha, and H. Y. Kim, Capillary rise of nonaqueous liquids in cellulose sponges, J. Fluid Mech. 818, R2 (2017).

[33] S. Chang, J. Seo, S. Hong, D. G. Lee, and W. Kim, Dynamics of liquid imbibition through paper with intra-fibre pores, J. Fluid Mech. 845, 36 (2018).

[34] Z. Sadjadi, M. Jung, R. Seemann, and H. Rieger, Meniscus arrest during capillary rise in asymmetric microfluidic pore junctions, Langmuir 31, 2600 (2015).

[35] H. Rieger, C. Thome, and Z. Sadjadi, Meniscus arrest dominated imbibition front roughening in porous media with elongated pores, J. Phys. Conf. Series 638, 012007 (2015).

[36] H. L. Frandsen, L. Damkilde, and S. Svensson, A revised multi-Fickian moisture transport model to describe non-Fickian effects in wood, Holzforschung 61, 563 (2007).

[37] J. Andersson, A. Ström, T. Gebäck, and A. Larsson, Dynamics of capillary transport in semi-solid channels, Soft Matter 13, 2562 (2017).

[38] D. P. Holmes, P. T. Brun, A. Pandey, and S. Protière, Rising beyond elastocapillarity, Soft Matter 12, 4886 (2016).
[39] C. Monteux, A. Tay, T. Narita, Y. De Wilde, and F. Lequeux, The role of hydration in the wetting of a soluble polymer, Soft Matter 5, 3713 (2009).

[40] A. Tay, C. Monteux, D. Bendejacq, and F. Lequeux, How a coating is hydrated ahead of the advancing contact line of a volatile solvent droplet. Eur. Phys. J. E 33, 203 (2010).

[41] A. Tay, D. Bendejacq, C. Monteux, and F. Lequeux, How does water wet a hydrosoluble substrate, Soft Matter 7, 6953 (2011).

[42] J. Crank, The Mathematics of Diffusion (Clarendon Press, Oxford, 1975).

[43] D. Mannes, W. Sonderegger, S. Hering, E. Lehmann, and P. Niemz, Non-destructive determination and quantification of diffusion processes in wood by means of neutron imaging, Holzforschung 63, 589 (2009).

[44] W. Olek, P. Perré, and J. Weres, Inverse analysis of the transient bound water diffusion in wood, Holzforschung 59, 38 (2005).

[45] I. N. Tsimpanogiannis, O. A. Moultos, L. F. M. Franco, M. B. de M. Spera, M. Erdös, and I. G. Economou, Self-diffusion coefficient of bulk and confined water: a critical review of classical molecular simulations studies, Molecul. Simul. 45, 425 (2019).

[46] A. King, N. Guignot, P. Zerbino et al., Tomography and imaging at the PSICHE beam line of the SOLEIL synchrotron, Rev. Sci. Instrum. 87, 093704 (2016)

[47] A. Mirone, E. Brun, E. Gouillart, P. Tafforeau, and J. Kieffer, The PyHST2 hybrid distributed code for high speed tomographic reconstruction with iterative reconstruction and a priori knowledge capabilities, Nucl. Instrum. Methods Phys. Res. Sect. B 324, 41 (2014).

[48] M. Levitt, P. Maduh, and C. Hughes, Cogwheel phase cycling, J. Magnetic Resonance 155, 300 (2002).

[49] R. S. Menon, A. L. Mackay, S. Flibotte, and J. R. T. Hailey, Quantitative separation of NMR images of water in wood on the basis of T2, J. Magnetic Resonance 82, 205 (1989). 